\title{
INSETOS CAPTURADOS COM ARMADILHA MALAISE NA ILHA DO MEL, BAÍA DE PARANAGUÁ, PARANÁ, BRASIL. I. COMPOSIC̣ÃO DE ORDENS
}

\author{
Renato R.C. Dutra ${ }^{2}$ \\ Renato C. Marinoni $^{2}$
}

\begin{abstract}
InSECTS CAPTIIRED WIIH Malaish trap at THE "IlHa dO MEL.", Baia de Paranagla. Parana. Brazil.. I. Oridnal composition. Two Malaise traps (TOWNES' 1972 model) were installed on an island (the "Ilha do Mel") in the Bay of Paranaguá, from Septemher. 1988 to August. 1989. at two sites denominated "Fortaleza" and "Praia Grande", for collection of insects. Apart from collection of insects (Fortaleza: 62.924 individuals: Praia Grande: 38,868 individuals), graphical analysis were liniled to those Orders wich occurred concomitantly at the two collection points in all months: Collembola. Hemiptera. Homoptera. Coleoptera. Lepidoptera. Diptera and Hymenoptera. It was concluded that the Malaise trap is selective for Diptera. Hymenoptera and Lepidoptera, and proved to be efticient in measuring abundance, in obtaining information regarding the seasonal variation of insects. and in comparing the entomofauna of different geographical localities.

KEY WORDS. Insect survey, Paraná, seasonal variation
\end{abstract}

Uma política de preservação ambiental deve contemplar a diversidade biológica com áreas representativas dos vários ambientes naturais ainda existentes. No caso do Estado do Paraná, que apresenta aproximadamente $5 \%$ de sua cobertura vegetal original (ITCF 1990), o conhecimento do potencial faunístico de diferentes áreas preservadas apresenta-se como fator fundamental para que se avalie suas condições como mantenedoras da biodiversidade, capaz de servir como estoque para repovoamentos (MARINONI \& DUTRA 1993).

Em agosto de 1986 teve início o Projeto "Levantamento da Fauna Entomológica do Estado do Paraná" (PROFAUPAR), sob a responsabilidade do Centro de Identificação de Insetos Fitófagos (CIIF) do Departamento de Zoologia da Universidade Federal do Paraná. Este Projeto visou ampliar o conhecimento da entomofauna paranaense, através da captura sistemática de insetos em oito localidades do Estado. Na área litorânea do Estado, o ponto de amostragem foi estabelecido em Antonina, na Reserva Biológica de Sapitanduva, Estrada Velha de Morretes a Antonina, em propriedade particular (MARINONi \& DUTRA 1993).

Apesar do ponto de amostragem em Antonina estar localizado na região litorânea, ele dista alguns quilômetros do litoral propriamente dito. A ação direta do mar faz-se mais branda a esta distância, levando a que florística e faunistica-

1) Contribuição número 790 do Departamento de Zoologgia. Universidade Federal do Paraná.

2) Departamento de Zoologia. Universidade Federal do Paraná. Caixa Postal 19()20, 81531-970 Curitiba, Paraná. Brasil. Bolsista do CNPy. 
mente se diferencie da Ilha do Mel (Baía de Paranaguá). Aproveitando o material já amostrado pelo PROFAUPAR e considerando as características florísticas diferenciadas da região litorânea, foram instaladas duas armadilhas Malaise na Ilha do $\mathrm{Mel}$, que permitiram a realização de um estudo comparativo de insetos capturados dentro de um mesmo ambiente e durante um mesmo período, além de possibilitar comparações entre áreas geograficamente próximas (Antonina e Ilha do $\mathrm{Mel}$ ), porém amostradas em períodos distintos.

\section{MATERIAL E MÉTODOS}

\section{DESCRIC̣̃o DA ÁREA DE ESTUdo}

Localizada na entrada da Baía de Paranaguá, entre a Ilha das Peças e Pontal do Sul (Fig. 1), a Ilha do Mel possui uma área total de 2762 hectares, dos quais 2585 hectares são de reserva ecológica. Possui um perímetro total de $35 \mathrm{Km}$ e a altitude máxima é de 151 metros (Boletim Informativo do Instituto de Terras, Cartografia e Florestas).

Pluviosidade (MAACK 1981): 1900-2000 mm/ano.

Temperatura média anual (MAACK 1981): $20-21^{\circ} \mathrm{C}$.

Classificação de Holdridge (MILANO et al. 1987): Transição Floresta úmida subtropical/muito úmida subtropical

Veloso \& Góes (1982): Floresta Ombrófila Densa Submontana

Classificação de Koeppen (MAACK 1981): Af (t)

As características geomorfológicas desta área podem ser encontradas em Bigarella (1946), Fernandes (1947), MaAck (1981) e Herrmann \& Rosa (in IBGE 1990).

Segundo MAACK (1981), quando a planície arenosa se eleva de 5 a 7,5 metros s.n.m., a estrutura xerofítica predomina em todas as plantas, com os componentes mais importantes pertencendo às famílias das mirtáceas, euforbiáceas, melastomatáceas, mirsináceas e cactáceas; a alternância entre restinga e mata costeira não se verificando apenas na região litorânea, mas também nas grandes ilhas: Peças, Pinheiros, Comprida e Ilha do Mel.

\section{LOCALIZAC̣ÃO DOS PONTOS DE AMOSTRAGEM E SUAS SITUAC̣ÕES FLORISTICAS}

$\mathrm{Na}$ Ilha do Mel foram escolhidos dois pontos de amostragem (Fig. 1) para instalação das armadilhas Malaise. O primeiro ponto "A " foi estabelecido no setor Norte (planície sedimentar quaternária, originada principalmente de depósitos marinhos) (FIgueIREDo 1954), próximo à Fortaleza de Nossa Senhora dos Prazeres, Reserva Ecológica com 2240 hectares, num ponto distando aproximadamente 400 metros da linha da maré mais alta, em um local menos sujeito à ação direta dos ventos.

Segundo SILVA (1990), como espécies importantes na floresta arenosa (região mais alta, com solo de maior drenagem) predominam: Ocotea pulchella (Ness) Mez, Clusia parviflora (Sald.) Engl., Ilex pseudobuxus Reiss., Ternstroemia brasiliensis Camb., Erytroxylum amplifolium (Mart.) Schult., Myrcia multiflora (Berg) Legr. e Psidium cattleianum Sabine. 


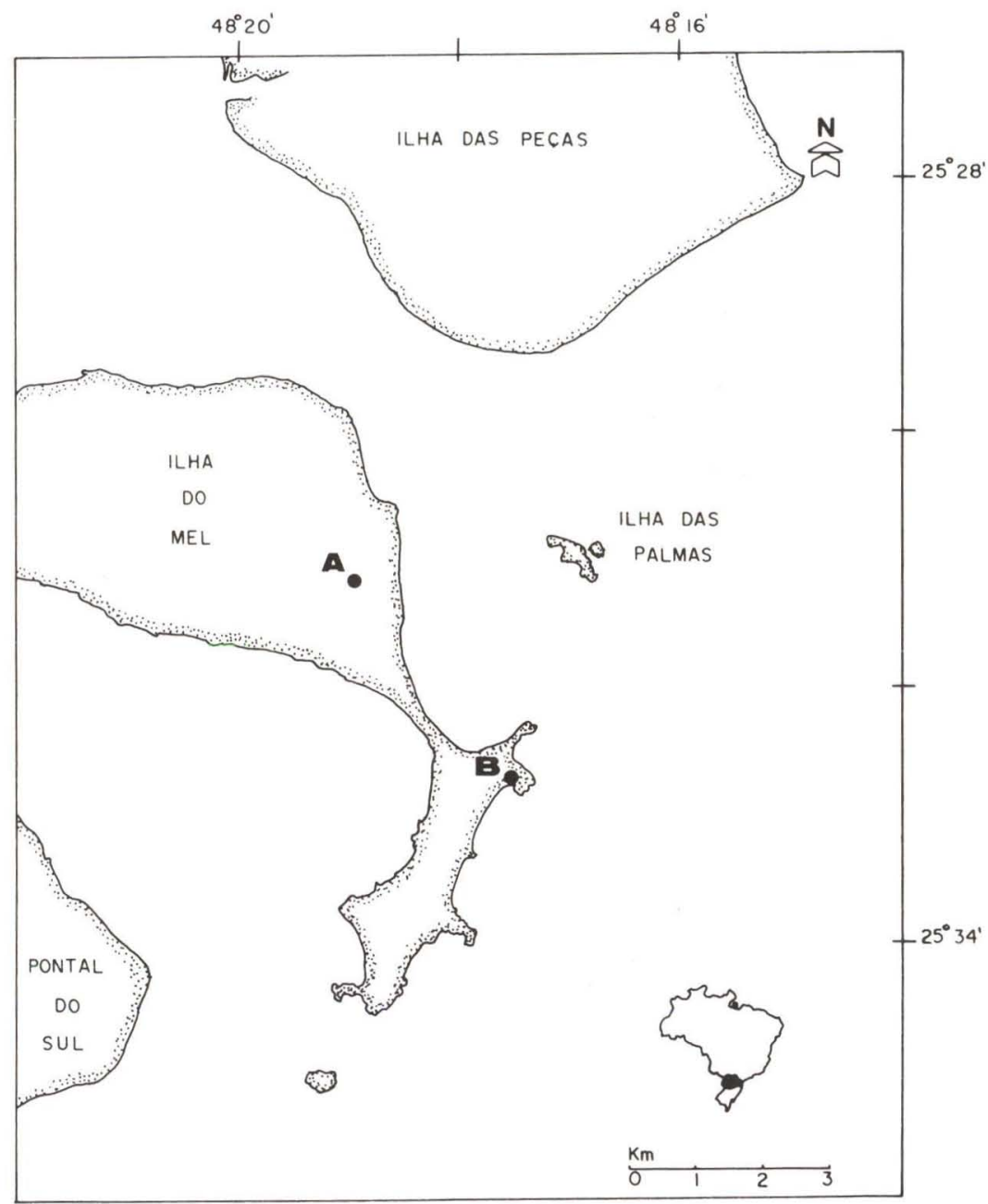

Fig. 1. Localização dos pontos amostrados na llha do Mol. com armadilha Malaise, no período de setembro de 1988 a agosto de 1989. Fortaleza (A) e Praia Grande (B).

O segundo ponto de amostragem "B" foi estabelecido no setor Sul (formado basicamente por afloramentos do complexo cristalino, de origem pré-cambriana, intercalados por pequenas planícies arenosas quaternárias) (FIGUEIREDO 1954), na Praia Grande (Reserva Natural com 345 hectares), bem na base do morro do Joaquim, em um local que no decorrer do trabalho mostrou-se sujeito a alagamento por vários dias, em decorrência da alta precipitação ocorrida em determinadas épocas. Distava aproximadamente 150 metros da linha da maior preamar e mostrava-se bastante exposto à ação dos ventos, se comparado ao outro ponto amostrado. 
Segundo MAACK (1981), os ventos vindos do setor Sul dominam com freqüência de $22,8 \%$ sobre os ventos vindos do setor Leste $(20,3 \%)$, e os ventos continentais dos setores SW, W e NW com freqüência de 33,2\%; a forte ação dos ventos oceânicos fazendo-se notar nas pitangueiras e aroeiras da praia, através da distribuição das suas folhas e na modificação do córtex de seus caules (FERNANDES 1947).

Segundo SiLVA (1990), como espécies importantes na floresta paludosa (que na época de maior pluviosidade permanece encharcada) destacam-se: Callophylum brasiliense Camb., Tabebuia cassinoides (Lam.) DC., Pouteria beaurepeirei (Glaz. \& Raunk.) Baeh., Myrcia grandiflora (Berg) Legr., Myrcia racemosa var. gaudichaudiana (Berg) Legr., Coussapoa microcarpa (Schott) Rizz., Marlierea tomentosa Camb. e Myrcia insularis Gardn.

\section{DADOS METEOROLÓgICOS}

Os dados meteorológicos utilizados (Estação meteorológica de Paranaguá) foram obtidos junto à Secretaria de Agricultura do Paraná.

\section{InStALAC̣Ão da MALAise E COLETA dAs AMOSTRAS OBTIDAS}

Em cada um dos pontos de amostragem foi instalada uma armadilha Malaise (modelo TownEs 1972), com as modificações no frasco-coletor propostas por Y AMAMOTO (1984). Os critérios utilizados para a definição do local e fixação da armadilha podem ser encontrados em MARINONI \& DUTRA (1993).

Todos os domingos, o material era retirado do frasco-coletor (contendo álcool hidratado a $70 \%$ ), e transferido para recipiente plástico devidamente rotulado (procedência e data de retirada).

As amostras foram agrupadas mensalmente, de acordo com a data de retirada do material do frasco-coletor, o que fez com que alguns meses tivessem cinco amostras enquanto outros apenas quatro (Tabs I e II); a primeira amostra datada de 04 de setembro de 1988 e a última de 27 de agosto de 1989, perfazendo um total de 52 semanas amostradas.

\section{TRIAGEM E ARMAZENAMENTO DOS DADOS OBTIDOS}

O material capturado foi triado a nível de Ordem em laboratório, sob microscópio estereoscópico, após o que foi acondicionado em recipientes de vidro (contendo álcool $70 \%$ ) devidamente rotulados que, por sua vez, foram guardados em recipientes plásticos (também contendo álcool 70\%) rotulados e codificados, para posterior acesso ao material.

Os dados coligidos foram transferidos para fichas (a seqüência utilizada para as Ordens foi aquela proposta por BORROR \& DELONG 1969) e posteriormente introduzidos em Banco de Dados, utilizando-se o programa DBase III.

\section{APRESENTAC̣Ão dos RESULTADOS E ANÁLISE GRÁFICA}

Somente as Ordens que ocorreram concomitantemente nos dois pontos amostrados, e durante todos os meses estudados, além do conjunto dos Insecta, tiveram suas flutuações da captura média (número médio de indivíduos/semana) analisadas graficamente. Também foi feita uma tentativa de visualização das 
relações entre os dados a partir das figuras feitas pelo método da Neográfica (=Graphique) e do tratamento gráfico da informação (BERTIN 1986). Este tratamento permite transformar gráficos "para ler" em gráficos "para ver" (CARDOSO 1984), substituindo gráficos ilustrativos por gráficos operacionais.

\section{MATERIAL-TESTEMUNHA}

Encontra-se depositado na Coleção de Entomologia "Pe. Jesus Santiago Moure", do Departamento de Zoologia da Universidade Federal do Paraná.

\section{RESULTADOS E DISCUSSÃO}

\section{CLIMA}

Fevereiro $\left(30,51^{\circ} \mathrm{C}-23,4^{\circ} \mathrm{C}\right.$ a $\left.36,0^{\circ} \mathrm{C}\right)$ foi o mês mais quente e julho $\left(13,06^{\circ} \mathrm{C}-6,8^{\circ} \mathrm{C}\right.$ a $\left.17,8^{\circ} \mathrm{C}\right)$ o mais frio; setembro $(85,63 \%-70,07 \%$ a $98,5 \%)$ foi o mês com a umidade relativa média mais alta e novembro $(67,84 \%-49,0 \%$ a $87,3 \%$ ) com a mais haixa. O mês de janeiro $(498,6 \mathrm{~mm})$ foi aquele com maior precipitação e agosto $(29,9 \mathrm{~mm})$ com a menor; apresentou um volume acumulado de $1931,8 \mathrm{~mm}$ ao final do período amostrado (Fig. 2).

A constatação de fevereiro e julho como os meses mais quente e frio, respectivamente, concorda com as observações feitas por DUQUIA \& CoSTA (1987), para a região litorânea do Estado, de que as temperaturas médias mais elevadas ocorrem no trimestre de janeiro a março, e as baixas de junho a agosto.

Quanto à umidade relativa média, um alto índice mensal durante todo o ano, com maior freqüência ocorrendo na faixa de $84 \%$ a $89 \%$ (DUQUIA \& COSTA, 1987), discorda dos resultados do presente trabalho, que constatou uma maior freqüência ocorrendo na faixa de $81 \%$ a $85 \%$ (seis meses), com cinco meses apresentando valores entre $75 \%$ e $79 \%$ e apenas um mês com valor abaixo de $70 \%$.

Segundo NIMER (in IBGE 1990), uma área que possui um regime anual de precipitação, cujo ritmo estacional é de certa forma regular, é representada pela faixa litorânea do Paraná e Santa Catarina; o máximo pluviométrico, nesta área restrita, pertencendo geralmente ao trimestre janeiro-fevereiro-março (DUQUIA \& Costa 1987), enquanto o mínimo incide, na maioria das vezes, no inverno, tratando-se portanto de um ritmo estacional característico das regiões de clima tropical.

Durante o período de amostragem, janeiro e agosto foram, respectivamente, os meses com maior e menor precipitação, concordando com os dados acima apresentados; o volume acumulado de $1.931,8 \mathrm{~mm}$, obtido ao final do período de coleta, também está relativamente próximo da média anual obtida por MAACK (1981) para a cidade de Paranaguá (região litorânea), que foi de $1.867,2 \mathrm{~mm}$.

\section{SOBRE O NÚMERO TOTAL DE INSECTA OBTIDO DURANTE O PERIODO AMOSTRADO}

Foram capturados 101.792 indivíduos nas duas localidades, sendo mais abundantes na Fortaleza (62.924 indivíduos - 61,82\% do total) (Tab. I). Na Praia Grande foram capturados 38.868 indivíduos, que representaram $38,18 \%$ do total de Insecta capturado na Ilha do Mel (Tab. II). 

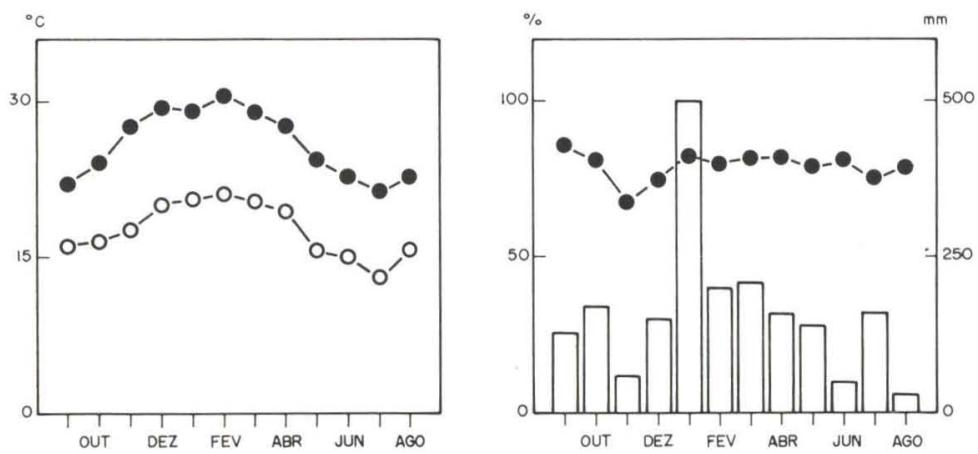

Fig. 2. Flutuação anual das temperaturas (médias) $\left({ }^{\circ} \mathrm{C}\right)$ mensais máxima $(\bullet)$ e mínima $(\mathbf{O})$, da umidade relativa (média) (\%) mensal $(\bullet)$ e da precipitação total $(\mathrm{mm})$ (barras verticais), no período de setembro de 1988 a agosto de 1989 .

O maior número de indivíduos capturados na Fortaleza, se comparada à Praia Grande, pode ser justificado de duas formas. Inicialmente, como foi observado por SILVA (1990), a região sujeita a inundações periódicas (Praia Grande no caso) apresenta árvores relativamente mais altas e com troncos mais grossos, assim como um espaçamento maior entre elas, enquanto a região livre destas inundações (Fortaleza) pode ser caracterizada por apresentar indivíduos menores, mais agrupados, sendo muitos destes bastante ramificados desde a sua base; as características estruturais desta última podendo favorecer a captura dos Insecta naquele estrato onde a ação da armadilha Malaise se faz presente (HUTCHENSON 1990). Estas mesmas características associadas ao fato da armadilha montada na Fortaleza estar mais no interior da mata do que aquela na Praia Grande, sugerem a maior exposição desta última à ação dos ventos, numa região onde os ventos vindos do setor Sul dominam sobre os ventos do setor Leste (MAACK 1981), podendo interferir na atividade de vôo dos Insecta (PRUESS \& PRUESS 1966).

\section{Presenca das Ordens}

Nas duas localidades, em conjunto, foram registradas 19 Ordens: Thysanura, Collembola, Ephemeroptera, Odonata, Orthoptera, Isoptera, Plecoptera, Dermaptera, Psocoptera, Thysanoptera, Hemiptera, Homoptera, Neuroptera, Coleoptera, Strepsiptera, Trichoptera, Lepidoptera, Diptera e Hymenoptera. Destas, Plecoptera e Dermaptera foram capturadas apenas na Fortaleza, enquanto Ephemeroptera e Trichoptera foram capturadas apenas na Praia Grande (Tabs I e II).

Na Fortaleza, Collembola, Orthoptera, Psocoptera, Hemiptera, Homoptera, Coleoptera, Lepidoptera, Diptera e Hymenoptera, foram as nove Ordens capturadas em todos os meses amostrados; Plecoptera e Dermaptera estiveram representadas em um único mês. Novembro e dezembro foram os meses em que foi registrado o maior número de Ordens: foi assinalada a presença de 15 das 17 Ordens encontradas nesta localidade, enquanto em junho e agosto apenas nove Ordens foram registradas (Fig. 3). 
Tabela I. Ilha do Mel. Fortaleza. Número total de indivíduos capturados com armadilha Malaise, por Ordem, e número de amostras por mês. no período de setembro de 1988 a agesto de 1989.

\begin{tabular}{|c|c|c|c|c|c|c|c|c|c|c|c|c|c|}
\hline ORDENS & SET (88) & OUT & NOV & DEZ & JAN (89) & FEV & MAR & $A B R$ & MA! & JUN & JUL & AGO & TOTAL \\
\hline THYSANURA & 0 & 0 & 1 & 0 & 0 & 2 & 0 & 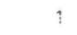 & 1 & 0 & 1 & 0 & 6 \\
\hline COLLEMBOLA & 51 & 67 & 42 & 41 & 64 & 37 & 28 & 123 & 60 & 33 & 31 & 28 & 605 \\
\hline EPHEMEROPTERA & 0 & 0 & 0 & 0 & 0 & 0 & 0 & 0 & 0 & 0 & 0 & 0 & 0 \\
\hline ODONATA & 0 & 0 & 0 & 2 & 0 & 0 & 1 & 1 & 0 & 0 & 0 & 0 & 4 \\
\hline ORTHOPTERA & 7 & 9 & 18 & 12 & 13 & 9 & 7 & 16 & 5 & 6 & $E$ & 5 & 12 \\
\hline ISOPTERA & 1 & 5 & 22 & 11 & 12 & 0 & 0 & 0 & 0 & 0 & 0 & 0 & 51 \\
\hline PLECOPTERA & 0 & 0 & 2 & 0 & 0 & 0 & 0 & 0 & 0 & 0 & 0 & 0 & 2 \\
\hline DERMAPTERA & 0 & 0 & 0 & 1 & 0 & 0 & 0 & 0 & 0 & 0 & 0 & 0 & $i$ \\
\hline PSOCOPTERA & 27 & 46 & 26 & 13 & 18 & 12 & 17 & 29 & 16 & 15 & 15 & 20 & 254 \\
\hline THYSANOPTERA & 4 & 2 & 2 & 1 & 3 & 0 & 1 & 0 & 1 & 0 & 0 & o & 14 \\
\hline HEMIPTERA & $z$ & 4 & 7 & $\mathrm{i}$ & 2 & 6 & 4 & 2 & 2 & 1 & 2 & 2 & 35 \\
\hline HOMOPTERA & 218 & 173 & 171 & 61 & 162 & 313 & 85 & 78 & 40 & 32 & 84 & 155 & 1572 \\
\hline NEUROPTERA & 2 & 3 & 2 & 1 & 1 & 2 & 2 & 0 & 0 & 0 & 0 & 0 & 13 \\
\hline COLEOPTERA & 40 & 104 & 195 & 136 & 111 & 95 & 101 & $i 15$ & 42 & 22 & 30 & 92 & 1083 \\
\hline STREPSIPTERA & 0 & 0 & 1 & 2 & e & 0 & 0 & 0 & 0 & 0 & 0 & 0 & 3 \\
\hline TRICHOPTERA & 0 & 0 & 0 & 0 & 0 & 0 & 0 & 0 & 0 & 0 & 0 & 0 & 0 \\
\hline LEPIDOPTERA & 172 & 470 & 608 & 537 & 446 & 337 & 260 & 339 & 110 & 66 & 99 & 161 & 3605 \\
\hline DIPTERA & 2357 & 6012 & 6012 & 1632 & 5712 & 5495 & 5179 & 9380 & 4413 & 1454 & 3282 & 2646 & 53574 \\
\hline HYMENOPTERA & 42 & 86 & 262 & 232 & 426 & 196 & 165 & 194 & 126 & 55 & 85 & 121 & 1990 \\
\hline TOTAL & 2923 & 698 & 7371 & 2683 & 6970 & 6504 & 5850 & 10278 & 4816 & 1684 & 3634 & 3230 & 62924 \\
\hline$N^{\circ}$ DE AMOSTRAS & 4 & 5 & 4 & 4 & 5 & 4 & 4 & 5 & 4 & 4 & 5 & 4 & 52 \\
\hline
\end{tabular}

Na Praia Grande, Collembola, Hemiptera, Homoptera, Coleoptera, Lepidoptera, Diptera e Hymenoptera, foram as sete Ordens capturadas em todos os meses amostrados; Trichoptera esteve representada em um único mês. Outubro e março foram os meses em que foi registrado o maior número de Ordens: foi assinalada a presença de 14 das 17 Ordens encontradas nesta localidade, enquanto em junho apenas sete Ordens foram registradas (Fig. 4).

\section{SOBREA FREQÜÊnCIA RELATIVA (\%) DAS ORDENS DE INSECTA MAIS ABUNDANTES, NOS DIFERENTES MESES DO ANO}

Na Fortaleza, as 6 Ordens mais abundantes foram Diptera (53.574 indivíduos), Lepidoptera (3.605), Hymenoptera (1.990), Homoptera (1.572), Coleoptera (1.083) e Collembola (605), perfazendo respectivamente $85,14 \%, 5,73 \%$, $3,16 \%, 2,50 \%, 1,72 \%$ e $0,96 \%$ do total de Insecta capturado; as 6 Ordens perfizeram $99,21 \%$ do total de Insecta capturado nesta localidade.

A variação da freqüência relativa dos Diptera foi de $60,83 \%$ a $91,63 \%$, registradas em dezembro e maio respectivamente. Para os Lepidoptera, foi de $2,28 \%$ (maio) a 20,01\% (dezembro); para os Hymenoptera, foi de 1,23\% (outubro) a $8,65 \%$ (dezembro); para os Homoptera, foi de $0,76 \%$ (abril) a $7,46 \%$ (setembro); para os Coleoptera, foi de $0,82 \%$ (julho) a $5.07 \%$ (dezembro) e para os Collembola, foi de $0,48 \%$ (março) a $1,96 \%$ (junho).

Na Praia Grande, as seis Ordens mais abundantes foram Diptera (28.074 
Tabela II. Ilha do Mel. Praia Grande. Número total de indivíduos capturados com armadilha Malaise, por Ordem, e número de amostras por mês, no período de setembro de 1988 a agosto de 1989.

\begin{tabular}{|c|c|c|c|c|c|c|c|c|c|c|c|c|c|}
\hline ORDENS & SET (88) & OUT & NOV & DEZ & JAN (89) & FEV & MAR & $A B R$ & MAI & JUN & JUL & AGO & TOTAL \\
\hline THYSANURA & 1 & 2 & 2 & 4 & 2 & 0 & 1 & 1 & 0 & 0 & 0 & 0 & 13 \\
\hline COLLEMBOLA & 171 & 363 & 542 & 768 & 172 & 12 & 5 & 10 & 3 & 14 & 11 & 20 & 2091 \\
\hline EPHEMEROPTERA & 0 & 1 & 0 & 0 & 0 & 0 & 2 & 3 & 0 & 0 & 0 & 0 & 1 \\
\hline ODONATA & 0 & 0 & 0 & 0 & 1 & 1 & 12 & 9 & 0 & 0 & 0 & 0 & 23 \\
\hline ORTHOPTERA & 2 & 6 & 11 & 44 & 29 & 8 & 7 & 13 & 1 & 0 & 1 & 0 & 122 \\
\hline ISOPTERA & 0 & 2 & 0 & 2 & 9 & 1 & 0 & 0 & 0 & 0 & 0 & 0 & 14 \\
\hline PLECOPTERA & 0 & 0 & 0 & 0 & 0 & 0 & 0 & 0 & 0 & 0 & 0 & 0 & 0 \\
\hline DERMAPTERA & 0 & 0 & 0 & 0 & 0 & 0 & 0 & 0 & 0 & 0 & 0 & 0 & 0 \\
\hline PSOCOPTERA & 39 & 81 & 43 & 46 & 25 & 16 & 9 & 5 & 2 & 0 & 5 & 4 & 275 \\
\hline THYSANOPTERA & 5 & 1 & 3 & 3 & 0 & 1 & 1 & 2 & 0 & 0 & 0 & 0 & 16 \\
\hline HEMIPTERA & 2 & 3 & 13 & 13 & 8 & 9 & 6 & 2 & 1 & 3 & 3 & 2 & 65 \\
\hline HOMOPTERA & 58 & 61 & 83 & 72 & 70 & 26 & 26 & 19 & 9 & 16 & 26 & 16 & 480 \\
\hline NEUROPTERA & 2 & 2 & 2 & 1 & 2 & 0 & 0 & 0 & 0 & 0 & 0 & 0 & 9 \\
\hline COLEOPTERA & 62 & 141 & 117 & 149 & 208 & 155 & 82 & 59 & 31 & 13 & 14 & 9 & 1030 \\
\hline STREPSIPTERA & 0 & 0 & 0 & 0 & 0 & 0 & 0 & 0 & 2 & 0 & 1 & 1 & 4 \\
\hline TRICHOPTERA & 0 & 0 & 0 & 0 & 0 & 0 & 1 & 0 & 0 & 0 & 0 & 0 & 1 \\
\hline LEPIDOPTERA & 238 & 926 & 1289 & 1159 & 476 & 365 & 299 & 278 & 86 & 60 & 93 & 10.7 & 5373 \\
\hline DIPTERA & 1748 & 6468 & 4779 & 2261 & 3599 & 3627 & 2079 & 1480 & 655 & 281 & 515 & 582 & 28074 \\
\hline HYMENOPTERA & 82 & 111 & 208 & 250 & 211 & 112 & 66 & 37 & 43 & 12 & 99 & 41 & 1272 \\
\hline TOTAL & 2400 & 8168 & 7092 & 4772 & 4812 & 4333 & 2596 & 1918 & 833 & 398 & 768 & 778 & 38868 \\
\hline$N^{\circ}$ DE AMOSTRAS & 4 & 5 & 4 & 4 & 5 & 4 & 4 & 5 & 4 & 4 & 5 & 4 & 52 \\
\hline
\end{tabular}

indivíduos), Lepidoptera (5.373), Collembola (2.091), Hymenoptera (1.272), Coleoptera (1.030) e Homoptera (480), perfazendo respectivamente $72,23 \%$, $13,82 \%, 5,38 \%, 3,27 \%, 2,65 \%$ e $1,23 \%$ do total de Insecta coletado; as 6 Ordens perfizeram $98,58 \%$ do total de Insecta coletado nesta localidade.

A variação da frequêencia relativa dos Diptera foi de $47,38 \%$ a $83,71 \%$, registradas em dezembro e fevereiro respectivamente. Para os Lepidoptera, foi de $8,42 \%$ (fevereiro) a $24,29 \%$ (dezembro); para os Collembola, foi de $0,19 \%$ (março) a 16,09\% (dezembro); para os Hymenoptera, foi de 1,36\% (outubro) a $12,89 \%$ (julho); para os Coleoptera, foi de 1,16\% (agosto) a 4,32\% (janeiro) e para os Homoptera, foi de $0,60 \%$ (fevereiro) a 3,77\% (junho).

A tabela III apresenta um quadro comparativo sumarizando os valores das freqüências percentuais dos Hemiptera, Homoptera, Coleoptera, Lepidoptera, Diptera e Hymenoptera obtidos no presente trabalho, acrescida dos resultados obtidos por MARSTON (1965), CANCELADO \& YONKE (1969), MATTHEWS \& Matthews (1970), Yamamoto (1984) e Marinoni \& Dutra (1993). Neste Quadro, fica evidente a semelhança nas freqüências percentuais dos grandes grupos, conforme observado por Y AMAMOTO (1984). 
$S \quad O N D J F \quad M A M \quad J \quad J \quad A$

THYSANURA

10

COLLEMBOLA

-

- $\bullet$

EPHEMEROPTERA

ODONATA

ORTHOPTERA

ISOPTERA

PLECOPTERA

O

DERMAPTERA

○

PSOCOPTERA

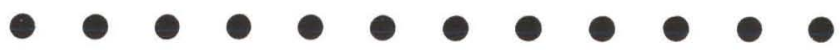

THYSANOPTERA

- $\bigcirc \circ$

-

HEMIPTERA

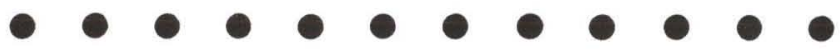

HOMOPTERA

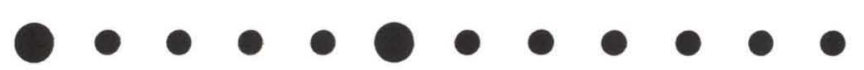

NEUROPTERA

- $\bullet \circ \circ$

COLEOPTERA

STREPSIPTERA

- $\bullet \bullet \bullet \bullet \bullet \bullet \bullet$

-

TRICHOPTERA

LEPIDOPTERA

DIPTERA

HYMENOPTERA
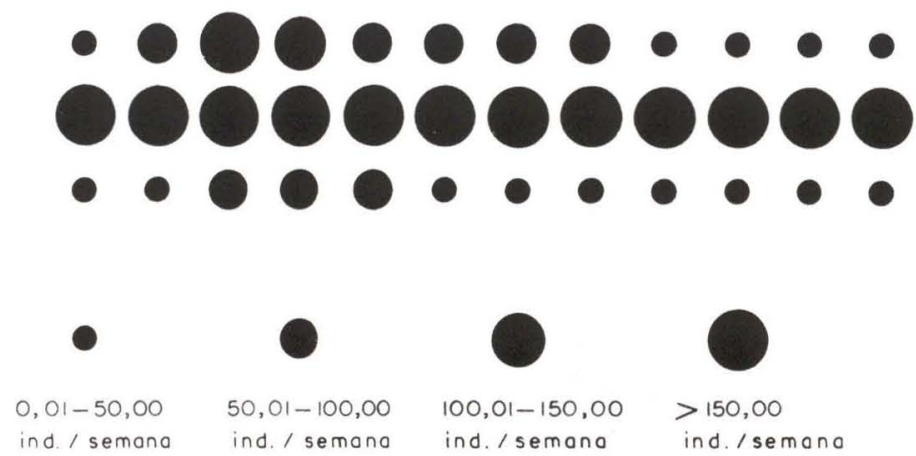

Fig. 3. Tha do Mel. Fortalesa. Captura meda (nemero medor de individues semana) das Ordens de Insecta. com armadilha Malaise. no periodo de setembro de 1988 a agosto de 1989. 


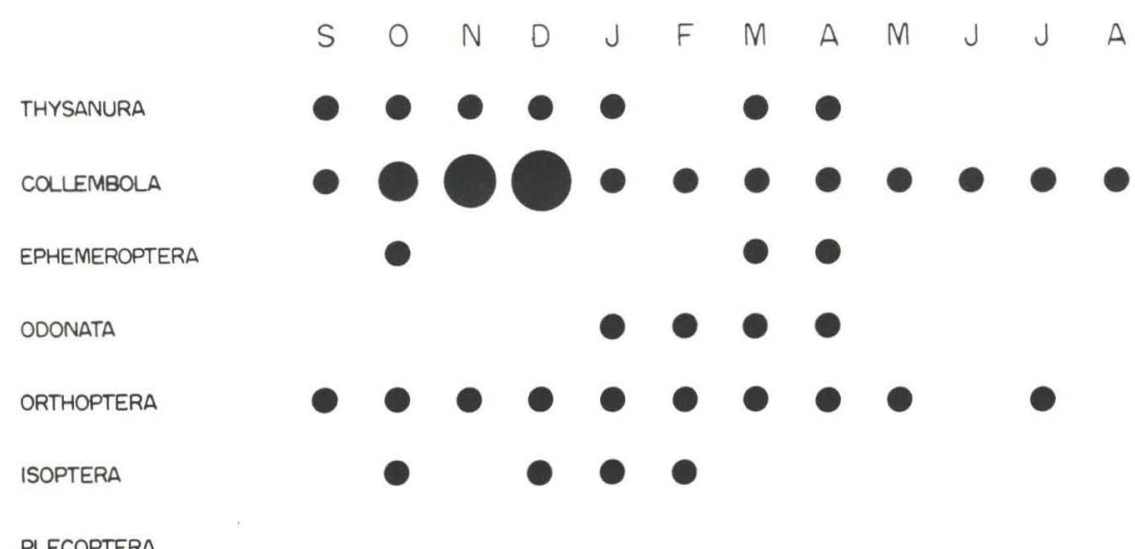

DERMAPTERA

PSOCOPTERA

THYSANOPTERA

HEMIPTERA

HOMOPTERA

NEUROPTERA

COLEOPTERA

STREPSIPTERA

TRICHOPTERA

LEPIDOPTERA

DIPTERA

HYMENOPTERA

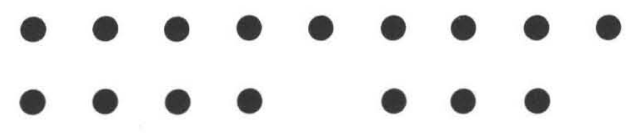

- 00000000

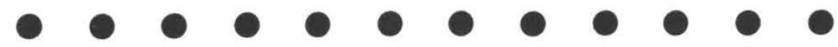

- $\bigcirc 0$

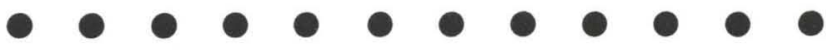

10

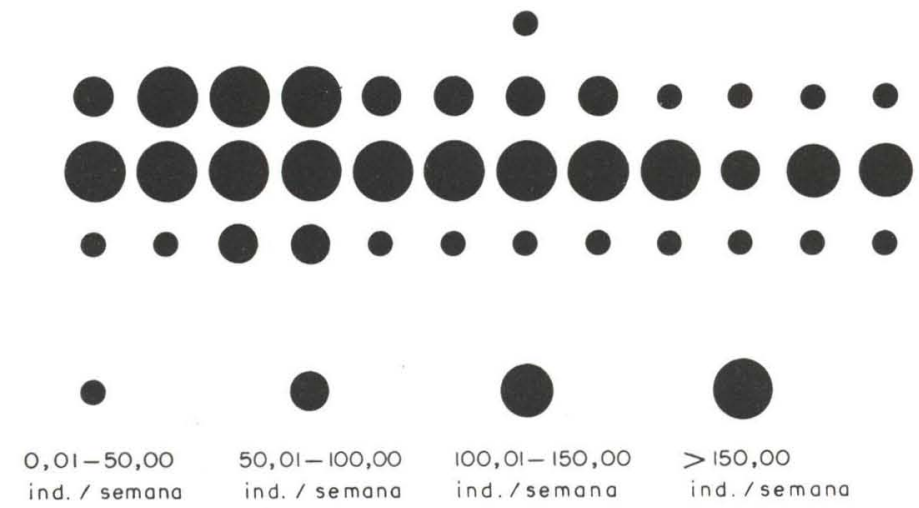

Fig. 4. Ilha do Mel. Praia Grande. Captura média (número médio de indivíduos/semana) das Ordens de Insecta, com armadilha Malaise, no período de setembro de 1988 a agosto de 1989. 
Tabela III. Quadro comparativo das freqüências relativas (\%) dos Hemiptera, Homoptera, Coleoptera, Lepidoptera, Diptera e Hymenoptera capturados em várias localidades do Paraná (Brasil) e dos Estados Unidos da América.

\begin{tabular}{|c|c|c|c|c|c|c|}
\hline LOCALIDADES & HEMIPTERA & HOMOPTERA & COLEOPTERA & LEPIDOPTERA & DIPTERA & HYMENOPTERA \\
\hline FORTALEZA & 0,1 & 2,5 & 1,7 & 5,7 & 85,1 & 3,2 \\
\hline PRAIA GRANDE & 0,2 & 1,2 & 2,7 & 13,8 & 72.2 & 3,3 \\
\hline KANSAS (USA) ' & 0,6 & 6,2 & 5,6 & 4,8 & 66,4 & 12,3 \\
\hline MISSOURI (USA) ${ }^{2}$ & 0.7 & 5,0 & 5,8 & 8,4 & 70,5 & 8,7 \\
\hline NEW YORK (USA) ${ }^{3}$ & & 6,9 & 2,4 & 7,2 & 45,0 & 14,7 \\
\hline CURITIBA URBANA ${ }^{4}$ & & 2,8 & 2,1 & 3,6 & 80,8 & 7,1 \\
\hline CURITIBA RURAL & - & 6,9 & 2,2 & 8,5 & 75,2 & 6.9 \\
\hline ANTONINA & 0,2 & 3,2 & 3,5 & 3,2 & 76,9 & 7,4 \\
\hline SÃO J. PINHAIS & 0,1 & 3,2 & 3,4 & 4,7 & 74,1 & 3,6 \\
\hline COLOMBO ${ }^{5}$ & 0,1 & 1,6 & 4,9 & 9,8 & 69,9 & 10.0 \\
\hline PONTA GROSSA & 0,1 & 1,4 & 2,0 & 2,7 & 87,1 & 5,4 \\
\hline GUARAPUAVA & 0,3 & 1,7 & 2,4 & 5,4 & 80,5 & 6,4 \\
\hline FÉNIX & 0.2 & 8,3 & 2,4 & 6,0 & 73,5 & 7,5 \\
\hline JUINDIAI DO SUL & 0,2 & 3.6 & 2,5 & 7,8 & 76,0 & 7,5 \\
\hline TELËMACO BORBA ${ }^{5}$ & 0.2 & 2,4 & 3,1 & 2,1 & 67,3 & 9,1 \\
\hline
\end{tabular}

Fontes: 1) Marston (1965: 156), 2) CAncelado \& Yonke (1969:85-86), 3) MatheWs \& MATHE:WS (1970: 57). 4) Yamamoto (1984: 21), 5) MARINONI \& DUTRA (1993:60).

\section{SOBRE A QUANTIDADE E A CAPTURA MÉDIA DO CONJUNTO DOS INSECTA E DE ALGUMAS ORDENS, NOS MESES AMOSTRADOS}

\section{A) CONJunto dos InSECTA}

$\mathrm{Na}$ Fortaleza, apresentaram uma variação da captura média de 421,00 a 2.055,60 indivíduos/semana, registradas em junho e abril respectivamente (Fig.5).

Na Praia Grande, apresentaram uma variação da captura média de 99,50 a 1.773,00 indivíduos/semana, registradas em junho e novembro respectivamente (Fig. 5).

Nas duas localidades, os menores valores da captura média foram registrados em junho, mês do trimestre em que ocorrem as temperaturas médias mais baixas da região (DUQUIA \& COSTA 1987), mostrando uma relação positiva entre as capturas médias e as baixas temperaturas. Quanto aos maiores valores da captura média, não foi possível estabelecer nenhuma relação com os dados climatológicos.

Quando comparamos os pontos amostrados na Ilha do Mel aqueles do PROFAUPAR (MARINONI \& DUTRA 1993), em função do número total de indivíduos capturados ao final do período amostrado, Antonina posiciona-se como intermediária entre a Fortaleza e a Praia Grande, sendo marcante a proximidade dos valores entre a Praia Grande (38.868 indivíduos) e São José dos Pinhais (39.224). Quando comparadas em função dos menores e maiores valores da captura média, e os meses em que foram registrados, novamente existem coincidências marcantes entre Praia Grande e São José dos Pinhais: as duas localidades apresentam os menores valores em junho e os maiores em novembro, e estes valores também estão relativamente próximos. Uma forma de explicar este fatoé estabelecer um paralelo entre as características climatológicas de São José dos 
238

DUTRA \& MARINONI
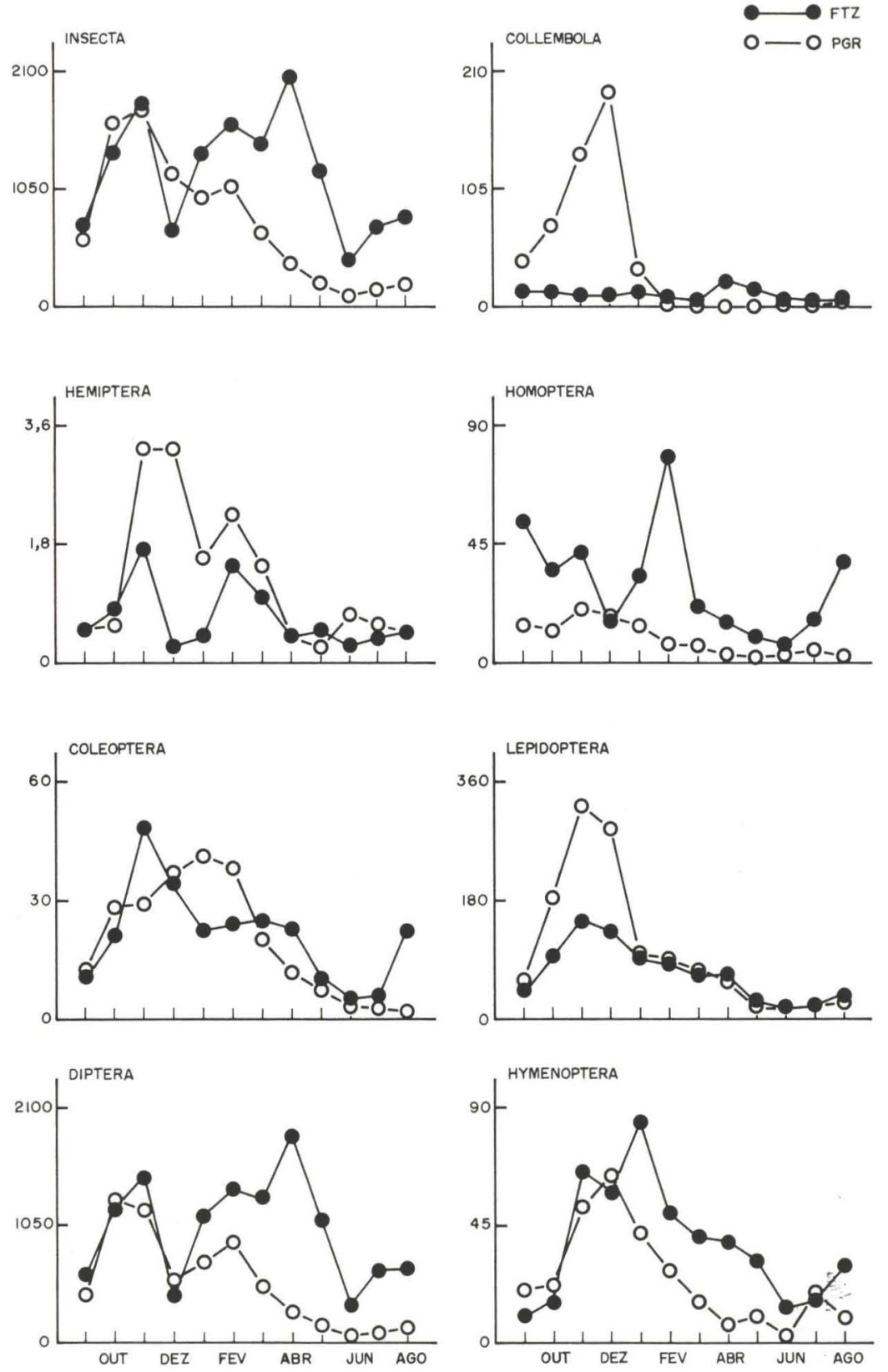

Fig. 5. Ilha do Mel. Flutuação anual da captura média (número médio de indivíduos/semana) do conjunto dos Insecta, Collembola, Hemiptera, Homoptera, Coleoptera, Lepidoptera, Diptera e Hymenoptera, com armadilha Malaise, no período de setembro de 1988 a agosto de 1989.

Revta bras. Zool. 11 (2): 227 - 245, 1994 
Pinhais e as características do local onde a armadilha está montada na Praia Grande, interferindo de maneira semelhante na atividade de vôo dos Insecta destas duas localidades.

Entre Fortaleza e Antonina chamam atenção a proximidade entre os valores (menores e maiores) da captura média e o mês de abril com uma captura média bastante alta nestas duas localidades.

B) Collembola

Na Fortaleza, apresentaram uma variação da captura média de 6,20 a 24,60 indivíduos/semana, registradas em julho e abril respectivamente (Fig. 5).

Na Praia Grande, apresentaram uma variação da captura média de 0,75 a 192,00 indivíduos/semana, registradas em maio e dezembro respectivamente (Fig.5).

A maior incidência de Collembola na Praia Grande deu-se exatamente quando do início das chuvas primavera, logo após um inverno normalmente seco, fazendo com que a partir de outubro, o local onde a armadilha estava montada, permanecesse totalmente encharcado por vários meses, chegando a apresentar uma lâmina d'água de aproximadamente $20 \mathrm{~cm}$ ao final de janeiro; esta relação entre o volume da precipitação e a quantidade de Collembola capturada concorda com as observaçōes de MATTHEws \& MATTHEws (1970), que também capturaram a maioria dos exemplares em dias chuvosos, quando as armadilhas estavam totalmente molhadas. A incidência de Collembola na Fortaleza sendo bem menor devido, provavelmente, ao fato deste local não ficar encharcado em momento algum.

Esta Ordem foi capturada nas oito localidades do PROFAUPAR, com a Fortaleza apresentando valores totais muito próximos daqueles obtidos para Fênix (616 indivíduos) e Colombo (623). O número de indivíduos capturados na Praia Grande foi bem próximo daquele registrado para Antonina (2.457) (MARINONI \& DUTRA 1993). Os valores percentuais confirmam as observações feitas com os valores totais, aproximando a Fortaleza $(0,96 \%)$ de Fênix $(0,8 \%)$ e a Praia Grande $(5,38 \%)$ de Antonina (4,7\%).

C) HEMIPTERA

$\mathrm{Na}$ Fortaleza, apresentaram uma variação da captura média de 0,25 a 1.75 indivíduos/semana, registradas em dezembro/junho e novembro respectivamente (Fig. 5)

Na Praia Grande, apresentaram uma variação da captura média de 0,25 a 3,25 indivíduos/semana, registradas em maio e novembro/dezembro respectivamente (Fig. 5).

Quando comparamos os valores totais de Hemiptera obtidos nas duas localidades da Ilha do Mel aqueles registrados durante o PROFAUPAR, a maior semelhança da Fortaleza é com Colombo. que também registrou 35 indivíduos, enquanto a Praia Grande é com São José dos Pinhais (MARINONI \& DuTRA 1993).

D) HOMOPTERA

Na Fortaleza, apresentaram uma variação da captura média de 8.00 a 78,25 
indivíduos/semana, registradas em junho e fevereiro respectivamente (Fig. 5).

Na Praia Grande, apresentaram uma variação da captura média de 2,25 a 20,75 indivíduos/semana, registradas em maio e novembro respectivamente (Fig.5).

Quando comparamos os valores totais de Homoptera obtidos nas duas localidades da Ilha do Mel, aqueles registrados durante o PROFAUPAR, é possível observar semelhanças entre a Fortaleza e Antonina, enquanto o valor da Praia Grande é próximo do valor obtido para Colombo (MARINONI \& DUTRA 1993).

\section{E) COLEOPTERA}

$\mathrm{Na}$ Fortaleza, apresentaram uma variação da captura média de 5,50 a 48,75 indivíduos/semana, registradas em junho e novembro respectivamente (Fig. 5).

Na Praia Grande, apresentaram uma variação da captura média de 2,25 a 41,60 indivíduos/semana, registradas em agosto e janeiro respectivamente (Fig.5).

Quando comparamos os valores totais de Coleoptera obtidos nas duas localidades da Ilha do Mel aqueles registrados durante o PROFAUPAR, observamos que a Fortaleza e a Praia Grande apresentaram valores próximos entre si e menores do que aqueles obtidos para todas as localidades do PROFAUPAR, estando mais próximos do valor obtido para Colombo (1.277 indivíduos) (MARINONI \& DUTRA 1993).

\section{F) LEPIDOPTERA}

Na Fortaleza, apresentaram uma variação da captura média de 16,50 a 152,00 indivíduos/semana, registradas em junho e novembro respectivamente (Fig. 5).

Na Praia Grande, apresentaram uma variação da captura média de 15,00 a 322,25 indivíduos/semana, registradas em junho e novembro respectivamente (Fig. 5).

É interessante notar que nas duas localidades da Ilha do Mel, as flutuações das capturas médias apresentaram tendências muito semelhantes, apesar dos valores totais e percentuais serem maiores na Praia Grande do que na Fortaleza.

As menores capturas médias foram registradas no inverno, demonstrando uma relação positiva com as baixas temperaturas. As maiores capturas médias ocorreram em novembro (fim da primavera/início do verão) e podem ser atribuídas à sincronização imposta ao desenvolvimento dos Insecta pela troca de estações, aparecimento de uma folhagem primaveril bastante fresca para oviposição e crescimento larval (MATTHEWS \& MATTHEWS 1970).

Quando comparamos os valores totais da Ilha do Mel àqueles obtidos durante o PROFAUPAR, a Fortaleza apresenta um valor próximo ao de Guarapuava (3.018 indivíduos), enquanto o valor da Praia Grande assemelha-se ao de Fênix (4.769) (MARINONI \& DUTRA 1993). Quando comparamos os valores percentuais, a relação entre a Fortaleza e Guarapuava é mantida, enquanto a Praia Grande apresenta um valor bem maior que as demais localidades (Tab. III), estando mais próximo do de Colombo $(9,8 \%)$. 


\section{G) DIPTERA}

Na Fortaleza, apresentaram uma variação da captura média de 363,50 a 1.876,00 indivíduos/semana, registradas em junho e abril respectivamente (Fig.5).

Na Praia Grande, apresentaram uma variação da captura média de 70,25 a 1.293,60 indivíduos/semana, registradas em junho e outubro respectivamente (Fig.5).

A freqüência percentual dos Diptera, nas duas localidades, confirmam a Seletividade da armadilha Malaise para esta Ordem (BREELAND \& PICKARD 1965; MatThews \& MatThews 1983).

Quando as flutuações das capturas médias são analisadas, verificamos que na Fortaleza o mês de abril foi aquele em que Diptera apresentou maior atividade, contrastando com a Praia Grande, que teve no mês de outubro o seu período de maior atividade; as duas localidades registrando a menor atividade no mês de junho.

Segundo ARAGÃo (1968), em trabalho realizado em diversas matas do município de Brusque (Santa Catarina), a curva da flutuação populacional dos Culicidae é um pouco atrasada em relação à da temperatura, com a atividade máxima verificando-se sempre em fevereiro ou março; tudo parecendo indicar que a população só vai atingir o máximo no outono, quando a queda da temperatura começa a freiar sua atividade.

Este fato explica, em parte, apesar da participação percentual de Culicidae não ter sido avaliada, a maior atividade dos Diptera, em abril, na Fortaleza. Não explica, porém, o porquê da queda na atividade que foi verificada na Praia Grande.

Uma análise mais detalhada das flutuações das capturas médias dos Diptera (Fig. 5), mostra que até o mês de março as tendências das mesmas, nas duas localidades, foram muito semelhantes, diferenciando-se em abril, para retornar a tendências semelhantes adiante. Anteriormente, quando abordamos a diferença entre o número total de indivíduos encontrados nas duas localidades, atribuímos à ação do vento uma grande importância, a qual parece salientar-se agora, visto que os Diptera praticamente determinaram as flutuações observadas para o conjunto dos Insecta.

A partir do mês de março, quando o fïnal do verão determina uma maior freqüência e intensidade dos ventos vindos do setor Sul, existe a possibilidade de migração dos Diptera das regiões mais expostas (Praia Grande no nosso caso) para o interior da mata, além da queda na atividade de vôo em decorrência destes ventos (PRUESS \& PRUESS 1966). A armadilha armada na Fortaleza, bem protegida no meio da mata, parece não sofrer a ação direta dos ventos, somando-se a isto a possibilidade de ter registrado o aumento da atividade populacional possível nesta época do ano (ARAGÃO 1968) e/ou provenientes das regiões mais expostas, até o mês seguinte, quando a queda da temperatura determina efetivamente a redução de sua atividade.

Quando comparamos os valores totais de Diptera obtidos nas duas localidades da Ilha do Mel aqueles registrados durante o PROFAUPAR, é possível observar semelhanças entre a Fortaleza e Fênix/Telêmaco Borba, enquanto o valor 
obtido na Praia Grande aproximam-na de São José dos Pinhais (MARINONI \& DUTRA 1993). Em função dos valores percentuais, a maior semelhança da Fortaleza é com Ponta Grossa, enquanto a Praia Grande mostra-se mais assemelhada à Fênix.

THYSANOPTERA
PSOCOPTERA
NEUROPTERA
ORTHOPTERA
ISOPTERA
PLECOPTERA
HEMIPTERA
COLEOPTERA
LEPIDOPTERA
ODONATA
DERMAPTERA
STREPSIPTERA
HYMENOPTERA
THYSANURA
HOMOPTERA
COLLEMBOLA
DIPTERA

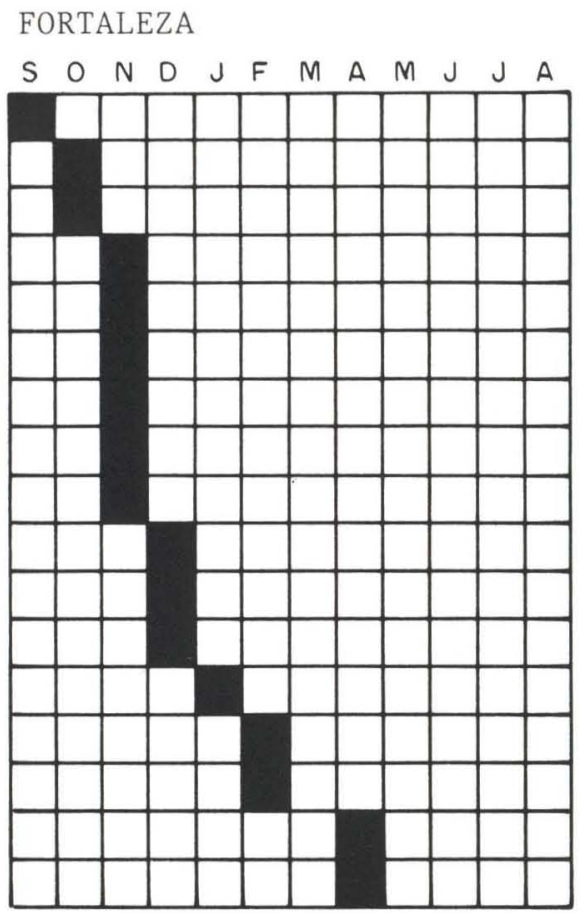

Fig. 6. Ilha do Mel. Representação neográfica dos meses em que as Ordens de Insecta, capturadas com armadilha Malaise, na Fortaleza, no período de setembro de 1988 a agosto de 1989, apresentaram sua maior atividade.

\section{H) HYMENOPTERA}

$\mathrm{Na}$ Fortaleza, apresentaram uma variação da captura média de 10,50 a 85,20 indivíduos/semana, registradas em setembro e janeiro respectivamente (Fig.5).

Na Praia Grande, apresentaram uma variação da captura média de 3,00 a 62,50 indivíduos/semana, registradas em junho e dezembro respectivamente (Fig.5).

ZANELLA (1991), estudando a estrutura da comunidade de abelhas da Ilha do Mel, capturadas com rede de varredura, registrou a maior atividade no final do mês de março e durante o mês de abril. No presente trabalho, utilizando outro método de captura, a maior atividade de Hymenoptera foi registrada, para a Fortaleza, no mês de janeiro, enquanto para a Praia Grande foi no mês de dezembro, sendo difícil estabelecer uma relação entre estes resultados. 
Quando comparamos os valores totais de Hymenoptera obtidos na Ilha do Mel aqueles registrados durante o PROFAUPAR (MARINONI \& DUTRA 1993), observamos que o valor da Fortaleza é intermediário àqueles obtidos para São José dos Pinhais (1.402 indivíduos) e Colombo (2610), enquanto a Praia Grande mostra-se mais assemelhada à São José dos Pinhais. Em termos percentuais, as duas localidades da Ilha do Mel aproximam-se de São José dos Pinhais $(3,6 \%)$ (Tab. III).

THYSANOPTERA
NEUROPTERA
PSOCOPTERA
DIPTERA
HOMOPTERA
LEPIDOPTERA
HEMIPTERA
THYSANURA
COLLEMBOLA
ORTHOPTERA
HYMENOPTERA
ISOPTERA
COLEOPTERA
ODONATA
TRICHOPTERA
EPHEMEROPTERA
STREPSIPTERA

\section{PRAIA GRANDE}

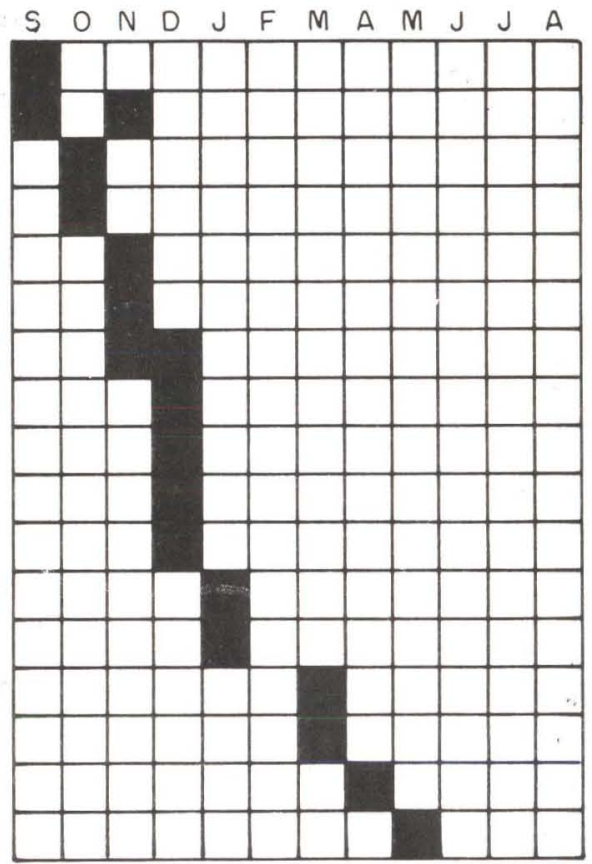

Fig. 7. Ilha do Mel. Representação neogrática dos meses em que as Ordens de Insecta. capturadas com armadilha Malaise, na Praia Grande, no período de setembro de 1988 a agosto de 1989. apresentaram sua maior atividade.

Finalmente, a representação neográfica mostra (Fig. 6) que, quando as Ordens de Insecta são dispostas na ordem cronológica dos meses em que apresentaram seus maiores valores de captura média, nove Ordens (Thysanoptera, Psocoptera, Neuroptera, Orthoptera, Isoptera, Plecoptera, Hemiptera, Coleoptera e Lepidoptera) tiveram seus maiores valores registrados na primavera; seis (Odonata, Dermaptera, Strepsiptera, Hymenoptera, Thysanura e Homoptera) no verão e apenas duas (Collembola e Diptera) no outono.

Na Praia Grande (Fig. 7), seis Ordens (Thysanoptera, Neuroptera, Psocoptera, Diptera, Homoptera e Lepidoptera) na primavera; seis (Thysanura, Collembola, Orthoptera, Hymenoptera, Isoptera e Coleoptera) no verão e quatro 
(Odonata, Trichoptera, Ephemeroptera e Strepsiptera) no outono. Hemiptera apresentou os mesmos valores máximos na primavera (novembro) e verão (dezembro).

\section{CONCLUSÕES}

Os resultados obtidos concordam com as observações de muitos autores quanto à capacidade seletiva da armadilha Malaise, notadamente para Diptera, Hymenoptera e Lepidoptera (JuILleT 1963; BREELAND \& PICKARD 1965; EvanS \& OWEN 1965; MATTHEWS \& MATTHEWs 1983), além de se mostrar eficiente para medir a abundância relativa (CHANTER 1965; EVANS \& OWEN 1965), na obtenção de informações sobre a variação sazonal dos insetos e comparação da entomofauna de diferentes localidades (CHANTER 1965; MARSTON 1965).

\section{REFERÊNCIAS BIBLIOGRÁFICAS}

Aragão, M.B. 1968. O ciclo anual de Anopheles do subgênero Kertezia, no Sul do Brasil. Mem. Inst. Oswaldo Cruz 66 (1): 85-106.

BERTIN, J. 1986. A neográfica e o tratamento gráfico da informação. Curitiba, Editora da Universidade Federal do Paraná, 273p.

Bigarella, J.J. 1946. Contribuição ao estudo da planície litorânea do Estado do Paraná. Arq. Biol. Tecnol. 1: 75-111.

Borror, D.J. \& D.M. Delong. 1969. Estudo dos Insetos. São Paulo, Ed. Edgard Blucher, 653p.

Breeland, S.G. \& E. Pickard. 1965. The Malaise trap - an efficient and unbiased mosquito collecting device. Mosquito News 25 (1): 19-21.

Cancelado, R. \& T.R. YonKe. 1969. Collecting prairie insects with malaise traps. Trans. Miss. Acad. Sc. 3: 83-88.

CARDoso, J.A. 1984. Construção de gráficos e linguagem visual. História: questões e debates 5 (8): 37-58.

CHANTER, D.O. 1965. The Malaise trap. Entomol. Record 77: 224-226.

DuQuia, C.G. \& T.V. CosTA. 1987. Comportamento médio das grandezas meteorológicas para a região litorânea do Estado do Paraná. Revta Floresta $17(1 / 2): 95-102$.

EVANS, F.C. \& D.F. OWEN. 1965. Measuring insect flight acti vity with a Malaise trap. Pap. Ac. Sc., Arts, and Lett. 50: 89-94.

Fernandes, L. 1947. Contribuição à Geografia da Praia de Leste. Arq. Mus. Paranaense 6: 3-44.

FigueIREDO, J.C. 1954. Contribuição à Geografia da Ilha do Mel (Litoral do Estado do Paraná). Tese de Cátedra, não publicada, Universidade Federal do Paraná, Curitiba, 61p.

HutChESON, J. 1990. Characterization of terrestrial insect communities using quantified, Malaise-trapped Coleoptera. Ecol. Entomol. 15: 143-151.

IBGE. 1990. Geografia do Brasil. Região Sul. Rio de Janeiro, Instituto Brasileiro 
de Geografia e Estatística, vol. 2, 420p.

ITCF. 1990. Atlas do Estado do Paraná. Curitiba, Instituto de Terras, Cartografia e Florestas, $\mathrm{XI}+73 \mathrm{p}$.

JUILLET, J.A. 1963. A comparasion of four types of traps used for capturing flying insects. Can. J. Zool. 41: 219-223.

MaAck, R. 1981. Geografia Física do Estado do Paraná. Rio de Janeiro, José Olympio Editora, 450p.

Marinoni, R.C. \& R.R.C. Dutra. 1993. Levantamento da fauna entomológica no Estado do Paraná. I. Introdução. Situação climática e florística dos oito pontos de coleta. Dados faunísticos de agosto de 1986 a julho de 1987. Revta bras. Zool. 8 (1-4): 31-73.

MARSTON, N. 1965. Recent modifications in the design of Malaise Insect traps with a summary of the insects represented in collections. J. Kansas Entomol. Soc. 38 (2): 154-162.

Matthews, R.W. \& J.R. MAtrhews. 1970. Malaise traps stu dies of flying insects in a New York mesic forest. I. Ordinal composition and Seasonal abundance. Jour. N.Y. Entomol. Soc. 78: 52-59.

Matthews, R.W. \& J.R. Matthews. 1983. Malaise traps. The Tow nes model catches more insects. Contrib. Amer. Ent. Inst. 20: 428-432.

Milano, M.S.; M.M. Brassiolo \& R.V. Soares. 1987. Zoneamento ecológico experimental do Estado do Paraná segundo o Sistema de Zonas de Vida de Holdridge. Revta Floresta 17 (1/2): 65-72.

PRUESS, K.P. \& N.C. PRUess. 1966. Note on a Malaise trap for determining flight direction of insects. J. Kansas Entomol. Soc. 39 (1): 98-102.

SILVA, S.M. 1990. Composição florística e fitossociologia de um trecho de Floresta de Restinga na Ilha do Mel, município de Paranaguá, PR. Tese de Mestrado, não publicada, Universidade Estadual de Campinas, Campinas, $146 \mathrm{p}$.

Townes, H. 1972. A light-weight Malaise trap. Ent. News 83: 239-247.

Veloso, P.H. \& L. GóEs Filho. 1982. Fitogeografia Brasileira. Classificação fisionômica-ecológica da vegetação neotropical. Bol. Téc. Projeto RADAMBRASIL, Série Vegetação, 85p.

Yamamoto, A.F. 1984. Fauna urbana e rural de Ichneumonidae (Hymenoptera) da região de Curitiba, Paraná. Tese de Mestrado, não publicada, Universidade Federal do Paraná, Curitiba, 116p.

Zanella, F.C.V. 1991. Estrutura da comunidade de abelhas silvestres (Hymenoptera, Apoidea) da Ilha do Mel, planície litorânea paranaense, Sul do Brasil. Tese de Mestrado, não publicada, Universidade Federal do Paraná, Curitiba, 88p.

Recebido em 10.I.1994; aceito em 26.IV.1994. 